\title{
ERRATUM AND ADDENDUM
}

(Received 11 February, 1970)

A. G. Gasanalizade: 'On the Physical Meaning of the Freundlich-Forbes Empirical Constant', Research Note in Solar Phys. 1 (1967), 482.

According to the statement at the end of the above mentioned 'Note', the numerical values of the Einstein gravitational red shift of the Lyman series limit of Hydrogen in the solar spectrum (constant $\lambda_{L \odot}$ ) and Freundlich-Forbes 'interaction displacement' (constant $Y$ ) would be slightly reduced. These additions are included in the corrections given below.

In Equation (5) we previously neglected the irreducible second term on the right, associated with the effect due to Earth's gravitational field. For correctness, however, we must add this value. Then, in the framework of Bohr's theory of hydrogen and hydrogen-like ions, Equation (5), should read as follows:

$$
\lambda_{L \odot}=\frac{G}{c^{2} R}\left(\frac{M_{\odot}}{R_{\odot}}-\frac{M_{\oplus}}{R_{\oplus}}\right)
$$

where $M_{\oplus}$ and $R_{\oplus}$ the Earth's mass and radius, respectively. After correction we find numerically

$$
\lambda_{L \odot}=1.93 \times 10^{-3} \AA .
$$

On the other hand, a mean value of the 'interaction displacement' $\langle Y\rangle$ would be within the range $Y_{1}<\langle Y\rangle<Y_{2}$, where

$$
Y_{1}=\frac{\sum^{14} Y_{1 j}}{14}=(1.78 \pm 0.03) \times 10^{-3} \AA \text {, }
$$

and

$$
Y_{2}=\frac{\sum^{14} Y_{2 j}}{14}=(2.04 \pm 0.04) \times 10^{-3} \AA
$$

This estimate, of course, is for the two values of $R_{\odot} / l_{0}$, being respectively 10000 and 100.

Previously we have confined the value $Y\left(=Y_{2}\right)$.

A calculation of the mean value of the 'interaction displacement' $\langle Y\rangle$ from Freundlich and Forbes's (1956) Tables IV and V give the following result:

$$
\langle Y\rangle=\frac{\sum^{14}\left(Y_{1 j}+Y_{2 j}\right) / 2}{14}=(1.92 \pm 0.06) \times 10^{-3} \AA,
$$

where $\pm 0.06 \times 10^{-3} \AA$ is the standard error. 
Thus we have again

$$
\langle Y\rangle \approx \lambda_{L \odot} .
$$

Shemakha Astrophysical Observatory of the Azerbaijan Academy of Sciences, Baku, U.S.S.R. 